\title{
The impact of primary tumor location in patients with metastatic colorectal cancer: a Korean Cancer Study Group COı2-04 study
}

Jae Ho Byun ${ }^{1}$, Joong Bae Ahn², Sun Young Kim³, Jung Hun Kang ${ }^{4}$, Dae Young Zang ${ }^{5}$, Seok Yun Kang ${ }^{6}$, Myoung Joo Kang ${ }^{7}$, Byoung Yong Shim ${ }^{8}$, Sun Kyung Baek ${ }^{9}$, Bong-Seog Kim ${ }^{10}$, Kyung Hee Lee ${ }^{11}$, Soon Il Lee ${ }^{12}$, Sang-Hee Cho ${ }^{13}$, Byeong Seok Sohn ${ }^{14}$, Samyong Kim ${ }^{15}$, In Gyu Hwang ${ }^{16}$, Eun Mi Nam ${ }^{17}$, Bong-Gun Seo ${ }^{18}$, Sang Cheul $\mathrm{Oh}^{19}$, Myung-Ah Lee ${ }^{20}$, Sang-Cheol Lee ${ }^{21}$, Ji Hyung Hong ${ }^{1}$, and Young Suk Park ${ }^{22}$

${ }^{1}$ Department of Internal Medicine, College of Medicine, Incheon St. Mary's Hospital, The Catholic University of Korea, Incheon; ${ }^{2}$ Division of Medical Oncology, Department of Internal Medicine, Yonsei University College of Medicine, Seoul; ${ }^{3}$ Center for Colorectal Cancer, National Cancer Center, Goyang; ${ }^{4}$ Department of Internal Medicine, Gyeongsang National University School of Medicine, Jinju; ${ }^{5}$ Department of Internal Medicine, Hallym University Sacred Heart Hospital, Anyang; ' ${ }^{6}$ Department of Hematology-Oncology, Ajou University School of Medicine, Suwon; ${ }^{7}$ Department of Hemato-Oncology, Inje University Haeundae Paik Hospital, Busan; ${ }^{8}$ Department of Internal Medicine, College of Medicine, St. Vincent's Hospital, The Catholic University of Korea, Suwon; ${ }^{9}$ Department of Internal Medicine, Kyung Hee University School of Medicine, Seoul; ${ }^{\circ}$ Department of Internal Medicine, Veterans Health Service Medical Center, Seoul; ${ }^{11}$ Division of Oncology-Hematology, Department of Medicine, Yeungnam University College of Medicine, Daegu; ${ }^{12}$ Department of Internal Medicine, Dankook University Hospital, Cheonan; ${ }^{13}$ Department of Hematology-Oncology, Chonnam National University Hwasun Hospital, Hwasun; ${ }^{14}$ Department of Internal Medicine, Inje University Sanggye Paik Hospital, Seoul; ${ }^{15}$ Department of Internal Medicine, Chungnam National University School of Medicine, Daejeon; ${ }^{16}$ Department of Internal Medicine, Chung-Ang University College of Medicine, Seoul; ${ }^{17}$ Department of Internal Medicine, Ewha Womans University School of Medicine, Seoul; ${ }^{18} \mathrm{De}-$ partment of Internal Medicine, Dongnam Institute of Radiological \& Medical Sciences, Busan; ${ }^{19}$ Division of Oncology-Hematology, Department of Internal Medicine, Korea University Guro Hospital, Seoul; ${ }^{20} \mathrm{De}-$ partment of Internal Medicine, College of Medicine, Seoul St. Mary's Hospital, The Catholic University of Korea, Seoul; ${ }^{21}$ Division of Hematology-Oncology, Department of Internal Medicine, Soonchunhyang University Cheonan Hospital, Cheonan; ${ }^{22}$ Division of Hematology-Oncology, Department of Medicine, Samsung Medical Center, Sungkyunkwan University School of Medicine, Seoul, Korea

Received: November 2, 2016

Revised : January 31, 2017

Accepted: May 17, 2017
Background/Aims: Colorectal cancer is associated with different anatomical, biological, and clinical characteristics. We determined the impact of the primary tumor location in patients with metastatic colorectal cancer (mCRC).

Methods: Demographic data and clinical information were collected from 1,115 patients from the Republic of Korea, who presented with mCRC between January 2009 and December 2011, using web-based electronic case report forms. Associations between the primary tumor location and the patient's clinical characteristics were assessed, and factors influencing overall survival were analyzed using Cox proportional hazards regression models.

Results: Of the 1,115 patients recruited to the study, 244 (21.9\%) had right colon cancer, $483(43.3 \%)$ had left colon cancer, and 388 (34.8\%) had rectal cancer. Liver and lung metastases occurred more frequently in patients with left colon and rectal cancer $(p=0.005$ and $p=0.006$, respectively), while peritoneal and ovarian metastases occurred more frequently in patients with right and left colon cancer $(p<0.001$ and $p=0.031$, respectively). The median overall survival of patients with tumors originating in the right colon was significantly shorter than that of patients whose tumors had originated in the left colon or rectum ( 13.7 months [95\% confidence interval (CI), 12.0 to 15.5 ] vs. 18.0 months [ $95 \%$ CI, 16.3 to 19.7 ] or 19.9 months [ $95 \% \mathrm{CI}, 18.5$ to 21.3 ], respectively; $p=0.003$ ). Tumor resection, the number of metastatic sites, and primary tumor location correlated with overall survival in the univariate and multivariate analyses.

Conclusions: Primary tumor location influences the metastatic sites and prognosis of patients with mCRC.

Keywords: Colorectal neoplasms; Primary tumor location; Survival

Correspondence to Young Suk Park, M.D.

Division of Hematology-Oncology, Department of Medicine, Samsung Medical Center, Sungkyunkwan University School of Medicine, 81 Irwon-ro, Gangnamgu, Seoul 06351, Korea

Tel: +82-2-3410-3454, Fax:+82-2-3410-1754, E-mail: pys27hmo@skku.edu 


\section{INTRODUCTION}

Colorectal cancer (CRC) represents the third and second most frequently diagnosed cancer in men and women, respectively. However, CRC mortality rates are high in a large number of countries worldwide [1]. In 2012, the Korea Central Cancer Registry contained 28,988 cases of newly diagnosed CRC. The crude incidence rate was an estimated 57.6 (69.3 for men and 45.9 for women) per 100,000 individuals, making CRC the third most frequently diagnosed cancer, after thyroid and gastric cancer [2].

CRC is associated with different anatomical, biological, and clinical characteristics. The subsite-specific incidences of CRC are approximately 25.0\%, 5.0\%, 5.0\%, $22.0 \%$, and $27.0 \%$ for ascending colon and cecum, transverse colon, descending colon, sigmoid colon, and the rectum, respectively [3]. Genetic and epigenetic alterations in CRC differ according to the primary tumor location [4]. CRCs associated with germline mutations of the APC gene develop in the distal colon in approximately $60.0 \%$ of the cases and in the rectum in approximately $25.0 \%$ of the cases. High microsatellite instability (MSI) and mismatch repair deficient colon cancers predominantly occur in the right colon $[5,6]$. Treatment for CRC also differs in the early stages. Rectal cancer requires radio- or chemoradiotherapy, whereas colon cancer does not [7]. However, the treatment for metastatic CRC (mCRC) is usually the same. A mCRC is treated with fluoropyrimidine and either irinotecan or oxaliplatin, which is associated with an increased overall survival (OS) of $>2.0$ years, with the addition of molecularly targeted agents [8]. Vascular endothelial growth factor (VEGF) inhibition with a monoclonal antibody, in combination with first- or second-line chemotherapy, has been shown to prolong survival in mCRC patients [9,10]. Monoclonal antibody inhibition of the epidermal growth factor receptor (EGFR) cetuximab or panitumumab has also been associated with improved patient outcomes, but only in the absence of RAS gene mutations [11,12].

A mCRC registry was designed to collect and evaluate data in patients with newly diagnosed primary mCRC. In this study, we aimed to evaluate the influence of the primary tumor location on the metastatic sites, KRAS gene mutation status, treatment patterns, and clinical outcomes in mCRC patients.

\section{METHODS}

This study was performed by the Colorectal Cancer Committee of the Korean Cancer Study Group (KCSG). The study was approved by the Institutional Review Board of KCSG (KCSG CO12-04) and each of the 22 participating institutions and performed in accordance with the principles of the Declaration of Helsinki. The informed consent was waived. The demographic data and clinical information of patients who were newly diagnosed with mCRC between January 2009 and December 2011 were reviewed using web-based electronic case report forms. Patients aged $\geq 18$ years who had pathologically confirmed adenocarcinoma with stage IV mCRC at initial diagnosis were enrolled in this study. Patients who had squamous cell carcinoma, non-Hodgkin's lymphoma, and neuroendocrine tumors (among others), or recurrences of early-stage CRC were excluded. Patient demographic data and information on primary tumor location, metastatic sites, treatment patterns, and clinical outcomes were collected by a physician or designated representative.

Primary tumor location was divided into the right colon, left colon, and the rectum. Tumors originating in the cecum, ascending colon, and proximal two-thirds of the transverse colon were categorized as cancers arising in the right colon. Tumors originating in the distal third of the transverse colon and sigmoid colon were categorized as cancers arising in the left colon. Because surgical resection of the primary $\mathrm{mCRC}$ had been performed in selected patients, the precise location of the primary tumor was difficult to ascertain, especially in the transverse colon, rectosigmoid colon, and upper rectum. Therefore, patients with transverse colon cancer were excluded and we arbitrarily defined rectal cancer as that originating in an approximate $15.0 \mathrm{~cm}$ region from the anal verge.

The KRAS gene mutations in this report refer to mutations in codon 12 or 13 in exon 2. KRAS genetic testing was performed using samples of paraffin-embedded tumor tissues that were sent to a laboratory for analysis using the established methods of each institution, including peptide nucleic acid-mediated real-time polymerase chain reaction clamping, direct sequencing, and pyrosequencing. 


\section{Statistical analyses}

The data were summarized using descriptive statistics. Continuous variables are represented by the frequency distribution, median, standard deviation, and extreme values. Categorical variables are represented by percentage frequency of each modality and 95\% confidence intervals (CIs). For categorical data, group comparisons were performed by an analysis of variance provided there was a reasonably normal distribution. Otherwise, chi-square or Fisher exact tests were performed. Progression-free survival (PFS) was estimated from the date of commencing treatment to the date of disease progression, or the date of death or last follow-up. OS was defined as the time from the date of pathological diagnosis to the date of death from any cause. PFS and OS were analyzed using the Kaplan-Meier method. For continuous data, analyses of variance tests were performed. A Cox proportional hazards regression model was used in the multivariate analysis of factors of OS that were significantly different in the univariate analysis. All statistical analyses were conducted using SPSS for Windows software version 19.0 (IBM Corp., Armonk, NY, USA). A p $<0.05$ was considered statistically significant.

\section{RESULTS}

\section{Patient characteristics}

In total, 1,166 patients were enrolled by 22 institutional members of the KCSG Colorectal Cancer Committee. Transverse colon cancers were difficult to divide into right and left colon cancers in the absence of surgery. Therefore, we excluded 51 patients with transverse colon cancer from this study. Of the remaining 1,115 patients, $685(61.4 \%)$ were men and 430 (38.6\%) were women. The baseline demographics and disease characteristics are summarized in Table 1. Seven hundred and twenty-seven patients (65.2\%) presented with metastatic colon cancer, including 244 patients (21.9\%) with right colon cancer, 483 patients $(43.3 \%)$ with left colon cancer, and 388 patients (34.8\%) with rectal cancer. The median age of all patients was 62 years (range, 31 to 94). The most common symptom of mCRC was abdominal pain, which occurred in 462 patients (41.4\%). However, 95 patients (8.5\%) were asymptomatic. Active treatment (surgical resection with or without chemotherapy, or chemotherapy alone) was performed in 1,003 patients (90.0\%). The remaining 112 patients received conservative care, such as symptomatic control of the intestinal obstruction by colostomy, stenting, palliative surgery, or radiation therapy.

\section{Metastatic patterns according to primary tumor lo- cation}

The most frequent metastatic sites of mCRC include the liver, lungs, extra-regional lymph nodes, and peritoneum. More than half of the patients (52.6\%) had a single metastatic site, in ascending order, in the liver (35.9\%), lungs (4.7\%), and peritoneum (4.3\%). The liver was the most frequent metastatic site (75.0\%), with or without other sites. Liver and lung metastases arose more frequently from primary left colon and rectal cancer than right colon cancer $(p=0.005$ and $p=0.006$, respectively). Peritoneal and ovarian metastases arose more frequently from primary right and left colon cancer than rectal cancer $(p<0.001$ and $p<0.05$, respectively) (Table 2 ). In contrast, no significant differences were observed between tumors originating from the right colon, left colon, or the rectum with respect to other rarer metastatic sites (e.g., the brain, bone, and spleen).

\section{Treatment for metastatic colorectal cancer}

The mCRC treatment did not differ according to primary tumor location. During this study, the use of anti-EGFR or anti-VEGF agents was limited due to the coverage of national insurance reimbursement. The majority of the patients were treated with sequential oxaliplatin-based or irinotecan-based combination chemotherapy, or an alternative sequence. The median number of chemotherapy regimens that were used was 2 (range, 1 to 7). One thousand and three patients (90.0\%) patients received first-line chemotherapy, and 728 patients (65.2\%) received second-line chemotherapy. The median number of cycles of chemotherapy for first- and second-line chemotherapy was 8 (range, 1 to 54 ) and 5 (range, 1 to 32 ), respectively (Table 3). Third-line chemotherapy was administered to 429 patients (38.5\%). Single-agent capecitabine was used in the majority (30.3\%) of these patients. In this study, $14.4 \%, 8.6 \%, 8.9 \%, 10.1 \%$, and $13.2 \%$ of patients who received first-, second-, third-, fourth-, and fifth-line chemotherapy, respectively, were involved in a clinical trial.

Signs or symptoms of an intestinal obstruction were 
Table 1. Baseline characteristics of patients with metastatic colorectal cancer

\begin{tabular}{|c|c|c|c|c|c|}
\hline \multirow{2}{*}{ Characteristic } & \multicolumn{4}{|c|}{ Primary tumor location } & \multirow{2}{*}{$p$ value } \\
\hline & $\operatorname{All}(\mathrm{n}=1,115)$ & Right colon $(n=244)$ & Left colon $(n=483)$ & $\operatorname{Rectum}(\mathrm{n}=388)$ & \\
\hline Age, yr & $62(31-94)$ & $66(31-94)$ & $62(31-88)$ & $60(32-87)$ & \\
\hline \multicolumn{6}{|l|}{ Sex } \\
\hline Male & $685(61.4)$ & $130(53 \cdot 3)$ & $289(59.8)$ & $266(68.6)$ & \\
\hline Female & $430(38.6)$ & $114(46.7)$ & $194(40.2)$ & $122(31.4)$ & $<0.001^{\mathrm{a}}$ \\
\hline \multicolumn{6}{|l|}{ Symptoms } \\
\hline Abdominal pain & $462(41.4)$ & $154(63.1)$ & $210(43.5)$ & $98(25 \cdot 3)$ & $<0.001^{\mathrm{a}}$ \\
\hline Bleeding & $316(28.3)$ & $22(9.0)$ & $118(24.4)$ & $176(55 \cdot 7)$ & $<0.001^{\mathrm{a}}$ \\
\hline Constipation & $223(20.0)$ & $27(11.1)$ & $107(22.2)$ & $89(22.9)$ & $<0.001^{\mathrm{a}}$ \\
\hline Weight loss & $142(12.7)$ & $39(16.0)$ & $52(10.8)$ & $51(13.1)$ & 0.131 \\
\hline Bowel habit change & $116(10.4)$ & $14(5 \cdot 7)$ & $46(9.5)$ & $56(14.4)$ & $0.002^{\mathrm{a}}$ \\
\hline Other ${ }^{b}$ & $169(15.2)$ & $38(15.6)$ & $73(15.1)$ & $58(14.9)$ & 0.977 \\
\hline Asymptomatic & $95(8.5)$ & $21(8.6)$ & $40(8.3)$ & $34(8.8)$ & 0.800 \\
\hline \multicolumn{6}{|l|}{ ECOG PS } \\
\hline 0 & $157(14.1)$ & $41(16.8)$ & $70(14.5)$ & $46(11.9)$ & \\
\hline 1 & $430(38.6)$ & $91(37 \cdot 3)$ & $163(33.7)$ & $176(45 \cdot 4)$ & \\
\hline 2 & $57(5.1)$ & $16(6.6)$ & $28(5.8)$ & $13(3.8)$ & \\
\hline$\geq 3$ & $18(1.6)$ & $3(1.2)$ & $12(2.5)$ & $3(0.8)$ & \\
\hline NA & $453(40.6)$ & $93(38.1)$ & $210(43 \cdot 5)$ & $150(38.7)$ & $0.023^{\mathrm{a}}$ \\
\hline \multicolumn{6}{|l|}{ Histological subtype } \\
\hline $\mathrm{ADC}$ & $1,089(97.7)$ & $230(94 \cdot 3)$ & $478(99.0)$ & $381(98.2)$ & \\
\hline MAC & $18(1.6)$ & $10(4.1)$ & $3(0.6)$ & $5(1.3)$ & \\
\hline SRCC & $8(0.7)$ & $4(1.6)$ & $2(0.4)$ & $2(0.5)$ & $0.011^{\mathrm{a}}$ \\
\hline \multicolumn{6}{|l|}{ Histology } \\
\hline Well differentiated & $212(19.0)$ & $37(15 \cdot 2)$ & $95(19.7)$ & $80(20.6)$ & \\
\hline Moderately differentiated & $693(62.2)$ & $144(59.0)$ & $311(64.4)$ & $238(34 \cdot 3)$ & \\
\hline Poorly differentiated & $126(11.3)$ & $36(14.8)$ & $46(9 \cdot 5)$ & $44(11.3)$ & \\
\hline Unknown & $61(5 \cdot 5)$ & $15(6.1)$ & $26(5 \cdot 4)$ & $20(5.2)$ & \\
\hline NA & $23(2.0)$ & $12(4 \cdot 9)$ & $5(1.0)$ & $6(1.5)$ & $0.010^{a}$ \\
\hline \multicolumn{6}{|l|}{ Metastatic sites } \\
\hline 1 & $586(52.6)$ & $130(53 \cdot 3)$ & $244(50.5)$ & $212(54.6)$ & \\
\hline 2 & $354(31.7)$ & $72(29.5)$ & $157(32.5)$ & $125(32.2)$ & \\
\hline$\geq 3$ & $175(15.7)$ & $42(17.2)$ & $82(17.0)$ & $51(13.1)$ & 0.448 \\
\hline
\end{tabular}

Values are presented as median (range) or number (\%).

ECOG, Eastern Cooperative Oncology Group; PS, performance status; NA, not available; ADC, adenocarcinoma; MAC, mucinous adenocarcinoma; SRCC, signet-ring cell carcinoma.

${ }^{\mathrm{a}} \mathrm{p}<0.05$

${ }^{\mathrm{b} A n a l}$ pain, low stool caliber, abdominal mass.

observed in 643 patients (57.7\%) (Table 1). Palliative or curative surgery, stenting, a combination of palliative or curative surgery and stenting, or an alternative method (e.g., conservative therapy, radiation therapy, chemo- therapy, or concurrent chemoradiotherapy) was used to treat the intestinal obstruction in 352 (31.6\%), 176 (15.8\%), $43(3.9 \%)$, and 72 patients $(6.4 \%)$, respectively. 
Table 2. Metastatic patterns according to the primary tumor location

\begin{tabular}{|c|c|c|c|c|c|}
\hline \multirow{2}{*}{ Metastatic site } & \multicolumn{4}{|c|}{ Primary tumor location } & \multirow{2}{*}{$p$ value } \\
\hline & $\operatorname{All}(\mathrm{n}=1,115)$ & Right colon $(n=244)$ & Left colon $(n=483)$ & $\operatorname{Rectum}(\mathrm{n}=388)$ & \\
\hline Liver & $836(75.0)$ & $170(69.7)$ & $385(79.7)$ & $281(72.4)$ & $0.005^{\mathrm{a}}$ \\
\hline Extra-regional lymph node & $347(31.1)$ & $78(32.0)$ & $145(30.0)$ & $124(32.0)$ & 0.786 \\
\hline Lung & $333(29.9)$ & $55(22.5)$ & $144(29.8)$ & $134(34 \cdot 5)$ & $0.006^{\mathrm{a}}$ \\
\hline Peritoneum & $180(16.1)$ & $70(28.7)$ & $79(16.4)$ & $31(8.0)$ & $<0.001^{\mathrm{a}}$ \\
\hline Bone & $77(6.9)$ & $11(4 \cdot 5)$ & $34(7.0)$ & $32(8.2)$ & 0.194 \\
\hline Ovary & $30(2.7)$ & $7(2.9)$ & $19(3.9)$ & $4(1.0)$ & $0.031^{\mathrm{a}}$ \\
\hline Brain & $9(0.8)$ & $1(0.4)$ & $4(0.8)$ & $4(1.0)$ & 0.695 \\
\hline
\end{tabular}

Values are presented as number (\%).

$\mathrm{a} p<0.05$.

Table 3. Characteristics of the first- and second- line chemotherapy regimen

\begin{tabular}{lcc}
\hline \multirow{2}{*}{ Characteristic } & \multicolumn{2}{c}{ Chemotherapy regimen } \\
\cline { 2 - 3 } & $\begin{array}{c}\text { First-line } \\
(\mathrm{n}=1,003)\end{array}$ & $\begin{array}{c}\text { Second-line } \\
(\mathrm{n}=728)\end{array}$ \\
\hline No. of cycles & $8(1-54)$ & $5(1-32)$ \\
$\leq 12$ & $866(86.3)$ & $668(91.8)$ \\
$>12$ & $137(13.7)$ & $60(8.2)$ \\
Chemotherapy regimen & & \\
Oxaliplatin-based regimen $^{\mathrm{a}}$ & $550(54.8)$ & $141(19.4)$ \\
Irinotecan-based regimen $^{\mathrm{b}}$ & $126(12.5)$ & $365(50.1)$ \\
Anti-EGFR agents $^{\mathrm{c}}$ & $41(4.1)$ & $30(4.1)$ \\
Anti-VEGF agents $^{\mathrm{d}}$ & $138(13.7)$ & $61(8.4)$ \\
Capecitabine & $57(5.7)$ & $48(6.6)$ \\
\hline Other & $93(9.2)$ & $83(11.4)$ \\
\hline
\end{tabular}

Values are presented as median (range) or number (\%).

EGFR, epidermal growth factor receptor; VEGF, vascular endothelial growth factor.

${ }^{a}$-Fluorouracil/leucovorin/oxaliplatin or capecitabine/oxaliplatin.

${ }_{5}$-Fluorouracil/leucovorin/irinotecan or capecitabine/irinotecan.

${ }^{\mathrm{c}}$ Cetuximab or panitumumab.

${ }^{\mathrm{d}}$ Bevacizumab.

\section{Oncological outcomes according to primary tumor location and chemotherapy with molecularly target- ed agents}

The median OS of all mCRC patients was 17.8 months (95\% CI, 16.7 to 18.9), although differences were observed according to primary tumor location (Table 4). The median OS of patients with tumors originating in the right colon was significantly shorter than that of patients whose tumors had originated in the left colon or rectum (13.7 months [ $95 \%$ CI, 12.0 to 15.5 ] vs. 18.0 months [ $95 \%$ CI, 16.3 to 19.7 ] or 19.9 months [ $95 \%$ CI, 18.5 to 21.3 ], respectively; $p=0.003$ ) (Fig. 1 ).

\section{Chemotherapy with molecularly targeted agents}

The majority of patients were treated with several chemotherapy regimens. Compared to patients who were not administered chemotherapy (i.e., receiving best supportive care only), a survival benefit was observed in the chemotherapy group, with a median OS of 19.2 (95\% CI, 18.1 to 20.3 ) months compared to 3.2 ( $95 \%$ CI, 1.5 to 4.9 ) months for the best supportive care group (Table 4). Despite anti-EGFR and anti-VEGF agents being restricted by the coverage of national insurance reimbursement, the use of anti-EGFR and anti-VEGF agents was associated with a prolonged survival. The median OS of patients who had received combination chemotherapy with anti-EGFR or anti-VEGF agents was significantly longer than that of patients who had received combination chemotherapy alone (22.0 months [ $95 \%$ CI, 19.5 to 24.5] vs. 18.5 months [ $95 \%$ CI, 17.2 to 19.7], respectively; $p<$ $0.05)$. This was particularly the case for patients with primary tumors originating from the left colon or rectum (Fig. 2). For patients treated with first-line chemotherapy, the median PFS was comparable to the median OS (7.0 months [ $95 \%$ CI, 6.5 to 7.5] vs. 8.9 months [ $95 \%$ CI, 8.1 to 9.8] for the chemotherapy alone and chemotherapy with molecularly targeted agent groups, respectively; $p$ $=0.002$ ). The effect of chemotherapy with molecularly targeted agents was more pronounced in left colon and 
Table 4. OS and PFS according to primary tumor location and treatment regimen

\begin{tabular}{|c|c|c|c|c|c|}
\hline \multirow[b]{2}{*}{ Characteristic } & \multicolumn{4}{|c|}{ Primary tumor location } & \multirow[b]{2}{*}{$p$ value } \\
\hline & $\begin{array}{c}\text { All } \\
(\mathrm{n}=1,115)\end{array}$ & $\begin{array}{l}\text { Right colon } \\
\quad(n=244)\end{array}$ & $\begin{array}{l}\text { Left colon } \\
(\mathrm{n}=483)\end{array}$ & $\begin{array}{l}\text { Rectum } \\
(\mathrm{n}=388)\end{array}$ & \\
\hline OS, mon & $17.8(16.7-18.9)$ & $13.7(12.0-15.5)$ & $18.0(16.3-19.7)$ & $19.9(18.5-21.3)$ & $0.003^{\mathrm{a}}$ \\
\hline Treatment regimen ${ }^{\mathrm{b}}$ & $19.2(18.1-20.3)$ & $16.2(14.0-18.3)$ & $19.4(17.9-21.0)$ & $20.8(19.5-22.1)$ & 0.086 \\
\hline Chemotherapy with targeted agents ${ }^{\mathrm{c}}$ & $22.0(19 \cdot 5-24.5)$ & $17.2(12.4-22.0)$ & $25.3(20.9-29.7)$ & $22.0(17.2-26.8)$ & $0.018^{\mathrm{a}}$ \\
\hline Chemotherapy without targeted agents & $18.5(17.2-19.7)$ & $15.6(13.1-18.1)$ & $17.9(15.8-20.0)$ & $20.1(18.8-21.4)$ & 0.166 \\
\hline Best supportive care & $3.2(1.5-4.9)$ & $2.5(0.4-4.6)$ & $5.4(2.2-8.6)$ & $2.8(0.0-6.5)$ & 0.122 \\
\hline \multicolumn{6}{|l|}{ PFS, mon } \\
\hline First-line chemotherapy & $7.6(7.1-8.0)$ & $7.4(6.6-8.2)$ & $7.8(7.2-8.3)$ & $7.5(6.8-8.2)$ & 0.265 \\
\hline Chemotherapy with targeted agents ${ }^{c}$ & $8.9(8.1-9.8)$ & $7 \cdot 5(5 \cdot 7-9 \cdot 3)$ & $9.5(8.4-10.7)$ & $8.7(6.8-10.5)$ & $0.023^{\mathrm{a}}$ \\
\hline Chemotherapy without targeted agents & $7.0(6.5-7.5)$ & $7.1(6.2-8.1)$ & $6.6(5 \cdot 9-7 \cdot 3)$ & $7.0(6.5-7.5)$ & 0.809 \\
\hline Second-line chemotherapy & $3 \cdot 7(3 \cdot 3-4 \cdot 0)$ & $3.5(2.7-4.2)$ & $3.6(3.0-4.2)$ & $3 \cdot 9(3.3-4 \cdot 0)$ & 0.225 \\
\hline Chemotherapy with targeted agents ${ }^{c}$ & $4.4(3.8-5.0)$ & $3.9(2.3-5 \cdot 4)$ & $4.8(3.9-5.7)$ & $4.1(3.0-5.2)$ & 0.092 \\
\hline Chemotherapy without targeted agents & $3 \cdot 7(3 \cdot 3-5 \cdot 0)$ & $3.9(2.3-5.4)$ & $4.8(3.9-5.7)$ & $4.1(3.0-5.2)$ & 0.712 \\
\hline
\end{tabular}

Values are presented as median (range).

OS, overall survival; PFS, progression-free survival.

${ }^{\mathrm{a}} \mathrm{p}<0.05$.

${ }^{b}$ Patients were treated by surgical resection with or without chemotherapy or chemotherapy alone.

${ }^{\mathrm{c}}$ Patients were treated by chemotherapy with anti-epidermal growth factor receptor and/or anti-vascular endothelial growth factor agents.

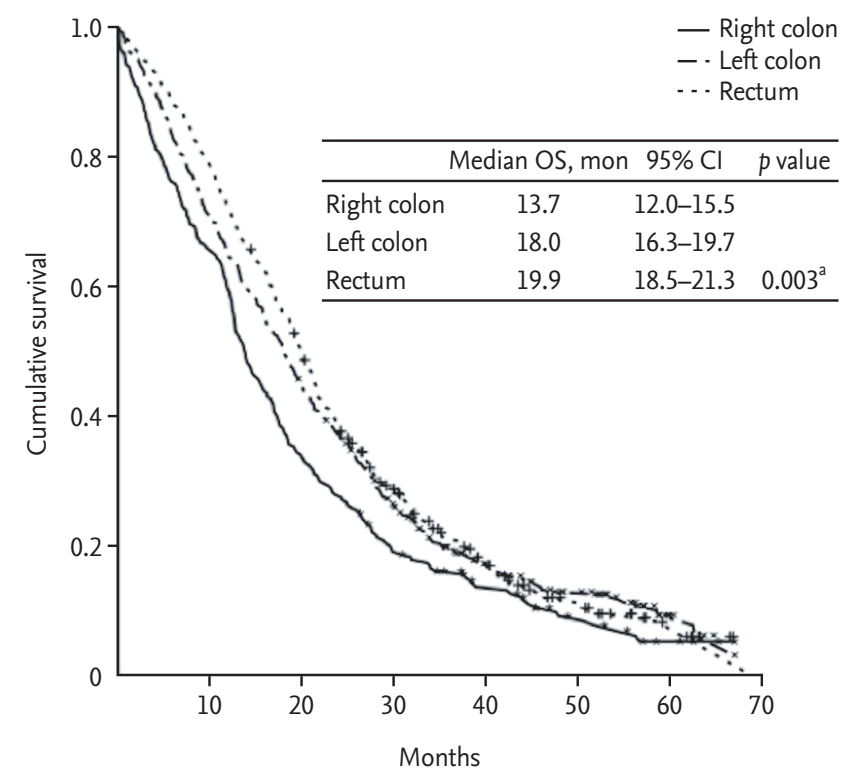

Figure 1. Kaplan-Meier curves of overall survival (OS) of patients with metastatic colorectal cancer according to primary tumor location. CI, confidence interval. ${ }^{a} p<0.05$.

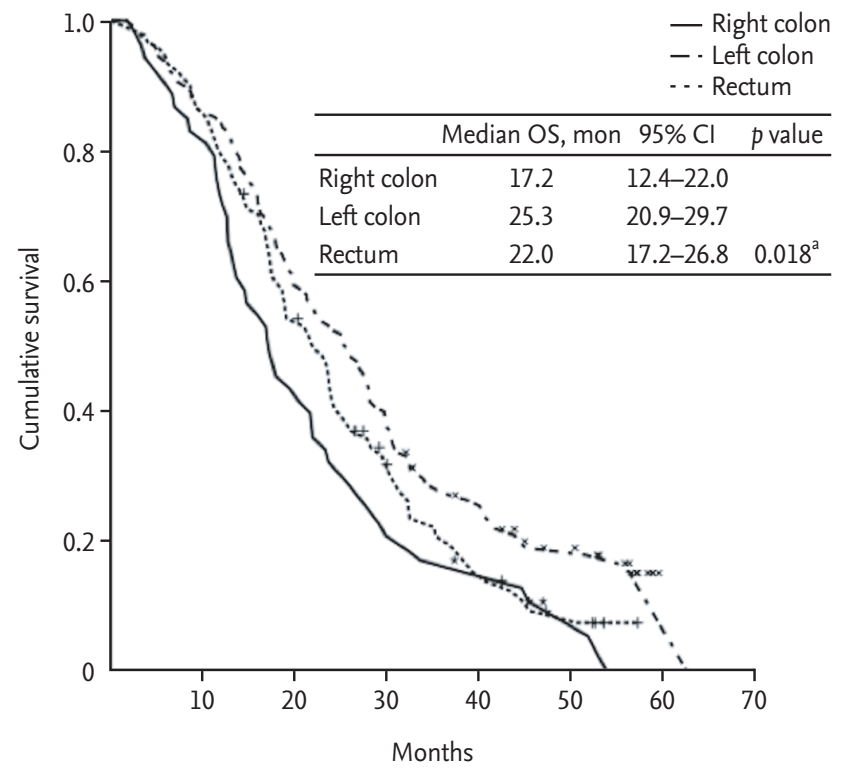

Figure 2. Kaplan-Meier curves of overall survival (OS) based on chemotherapy with molecularly targeted agents according to primary tumor location. CI, confidence interval. ${ }^{a} p<0.05$. 
rectal cancer patients than right colon cancer patients $(p$ $<0.05$ ). However, for patients treated with second-line chemotherapy, the effect of molecularly targeted agents was reduced and no significant differences were observed with respect to primary tumor location.

\section{KRAS mutation status and primary tumor location}

The KRAS genetic testing was performed in 516 of the 1,115 patients (46.3\%) included in this study. At the time, only exon 2 was screened due to the definition of a wildtype KRAS gene. KRAS genetic testing was performed by peptide nucleic acid-mediated real-time polymerase chain reaction clamping, direct sequencing, or pyrosequencing. The mutant KRAS gene was detected in 179 patients (34.7\%) and was more frequently observed in patients with primary right colon cancer $(p=0.004)$ (Table 5). Codon 12 mutations were detected in 132 patients (73.7\%) and codon 13 mutations were detected in 42 patients (23.5\%). The KRAS mutation status was unknown for the remaining five patients $(2.8 \%)$. In this study, the KRAS mutation status itself did not influence the median OS. However, in patients with the wild-type KRAS gene, chemotherapy with molecularly targeted agents and the primary tumor location correlated with OS (Ta-

Table 5. KRAS mutation status according to primary tumor location

\begin{tabular}{|c|c|c|c|c|c|}
\hline & & Primary & location & & b \\
\hline & All & Right colon & Left colon & Rectum & Praiue \\
\hline KRAS mutation status & 516 & 100 & 246 & 170 & \\
\hline Wild-type & $337(65 \cdot 3)$ & $51(51.0)$ & $170(69.1)$ & $116(68.2)$ & \\
\hline Mutant & $179(34.7)$ & $49(49.0)$ & $76(30.9)$ & $54(31.8)$ & $0.004^{a}$ \\
\hline KRAS mutation site & 179 & 49 & 76 & 54 & \\
\hline Codon 12 & $132(73.7)$ & $34(69.4)$ & $58(73.6)$ & $40(74.1)$ & \\
\hline Codon 13 & $42(23 \cdot 5)$ & $14(28.6)$ & $16(21.1)$ & $12(22.2)$ & \\
\hline Unknown & $5(2.8)$ & $1(2.0)$ & $2(2.6)$ & $2(3.7)$ & 0.873 \\
\hline
\end{tabular}

Values are presented as number (\%).

${ }^{a} p<0.05$.

Table 6. OS and PFS according to primary tumor location and KRAS mutation status

\begin{tabular}{|c|c|c|c|c|c|c|}
\hline \multirow[b]{2}{*}{ Characteristic } & \multicolumn{5}{|c|}{ Primary tumor location } & \multirow[b]{2}{*}{$p$ value } \\
\hline & $\begin{array}{c}\text { All } \\
(n=516)\end{array}$ & $p$ value & $\begin{array}{l}\text { Right colon } \\
\quad(n=100)\end{array}$ & $\begin{array}{l}\text { Left colon } \\
(\mathrm{n}=246)\end{array}$ & $\begin{array}{l}\text { Rectum } \\
(\mathrm{n}=170)\end{array}$ & \\
\hline \multicolumn{7}{|l|}{ OS, mon } \\
\hline Wild-type KRAS & $20.6(18.5-22.7)$ & 0.310 & $15.6(12.6-18.5)$ & $21.7(17.6-25.8)$ & $21.6(18.6-22.7)$ & $0.008^{\mathrm{a}}$ \\
\hline Mutant KRAS & $20.7(18.7-22.8)$ & & $17.5(13.2-21.8)$ & $20.8(18.0-23.6)$ & $21.0(15.6-26.4)$ & 0.689 \\
\hline \multicolumn{7}{|c|}{ First-line chemotherapy } \\
\hline Overall PFS, mon & $7.6(7.2-8.0)$ & & $7.4(6.6-8.2)$ & $7.8(7.2-8.3)$ & $7 \cdot 5(6.8-8.0)$ & 0.265 \\
\hline Wild-type KRAS & $8.2(7.5-8.9)$ & & $7 \cdot 4(5 \cdot 0-9 \cdot 7)$ & $8.6(7 \cdot 9-9 \cdot 3)$ & $8.5(7.3-9.7)$ & 0.077 \\
\hline Mutant KRAS & $8.3(7.5-9.2)$ & 0.692 & $8.3(6.4-10.3)$ & $7.8(6.1-9.5)$ & $8.0(6.4-9.6)$ & 0.892 \\
\hline \multicolumn{7}{|c|}{ Second-line chemotherapy } \\
\hline PFS, mon & $3.7(3 \cdot 3-4.0)$ & & $3.5(2.7-4.2)$ & $3.6(3.0-4.2)$ & $3.9(3.3-4.5)$ & 0.225 \\
\hline Wild-type KRAS & $4.1(3 \cdot 5-4.7)$ & & $3.1(2.0-4.2)$ & $3.8(2.7-4.9)$ & $4.8(3.5-4.7)$ & $0.044^{\mathrm{a}}$ \\
\hline Mutant KRAS & $3.5(2.8-4.2)$ & 0.259 & $3.7(2.4-5.0)$ & $2.6(1.4-3.7)$ & $3.7(2.7-4.7)$ & 0.223 \\
\hline
\end{tabular}

Values are presented as median (range).

OS, overall survival; PFS, progression-free survival.

${ }^{\mathrm{a}} \mathrm{p}<0.05$. 
bles 6 and 7). Patients with primary left colon or rectal tumors that expressed the wild-type KRAS gene had a significant longer median OS than patients with primary right colon tumors (21.7 months [ $95 \% \mathrm{CI}, 17.6$ to 25.8 ] or 21.6 months [ $95 \% \mathrm{CI}, 18.6$ to 22.7 ] vs. 15.6 months [ $95 \%$ CI, 12.6 to 18.5 ], respectively; $p=0.008$ ).

\section{Surgical resection and primary tumor location}

Surgical resection of the primary and metastatic tumors was associated with a prolonged survival. Curative resections were performed in 218 patients (19.6\%). One hundred and thirty-eight patients (12.4\%) underwent surgical resection as the initial treatment and 80 patients (7.2\%) underwent surgical resection during first- or second-line chemotherapy. Curative resections were usually performed by resecting the primary tumor and metastatic tumors with or without radiofrequency ablation. Palliative resections were performed in 330 patients (29.6\%). The majority of palliative resections were primary tumor resections (Table 8). Irrespective of whether the surgical resections were curative, tumor resection was associated with a more favorable prognosis. The median OS was 38.9 months ( $95 \%$ CI, 34.0 to 43.8 ) for patients who had undergone curative resection, 19.7 months (95\% CI, 18.0 to 21.4) for patients who had undergone palliative resection, and 12.1 months $(95 \% \mathrm{CI}$, 11.2 to 13.1) for patients who did not undergo surgical resection $(p=0.007)$. However, in patients who underwent

Table 7. OS and PFS according to chemotherapy regimen and KRAS mutation status

\begin{tabular}{|c|c|c|c|}
\hline \multirow{2}{*}{ Characteristic } & \multicolumn{2}{|c|}{ Chemotherapy } & \multirow{2}{*}{$p$ value } \\
\hline & With targeted agents ${ }^{\mathrm{a}}$ & Without targeted agents & \\
\hline \multicolumn{4}{|l|}{ OS, mon } \\
\hline Wild-type KRAS & $24.0(20.6-26.7)$ & $18.9(17.2-20.7)$ & $0.015^{b}$ \\
\hline Mutant KRAS & $21.8(16.8-26.7)$ & $20.1(18.1-22.1)$ & 0.670 \\
\hline \multicolumn{4}{|l|}{ PFS, mon } \\
\hline \multicolumn{4}{|c|}{ First-line chemotherapy } \\
\hline Wild-type KRAS & $9.0(7.9-10.1)$ & $7.6(6.4-8.7)$ & 0.063 \\
\hline Mutant KRAS & $9.1(7.6-10.7)$ & $7.8(6.3-9.2)$ & 0.093 \\
\hline \multicolumn{4}{|c|}{ Second-line chemotherapy } \\
\hline Wild-type KRAS & $4.6(3.7-5 \cdot 5)$ & $3.5(2.6-4.6)$ & 0.128 \\
\hline Mutant KRAS & $4.4(2.7-4.2)$ & $3.1(2.3-4.0)$ & $0.006^{\mathrm{b}}$ \\
\hline
\end{tabular}

Values are presented as median (range).

OS, overall survival; PFS, progression-free survival.

${ }^{a}$ Anti-epidermal growth factor receptor and anti-vascular endothelial growth factor agents.

${ }^{\mathrm{b}} \mathrm{p}<0.05$.

Table 8. Surgical outcome according to the primary tumor location

\begin{tabular}{|c|c|c|c|c|c|}
\hline \multirow{2}{*}{ Characteristic } & \multicolumn{4}{|c|}{ Primary tumor location } & \multirow{2}{*}{$p$ value } \\
\hline & All $(n=548)$ & Right colon $(n=123)$ & Left colon $(n=235)$ & $\operatorname{Rectum}(\mathrm{n}=190)$ & \\
\hline Curative surgery & $218(19.6)$ & $36(16.5)$ & $93(42.7)$ & $89(40.8)$ & \\
\hline Palliative surgery & $330(29.6)$ & $87(26.4)$ & $142(43.0)$ & $101(30.6)$ & \\
\hline \multicolumn{6}{|l|}{ OS, mon } \\
\hline Curative surgery & $38.9(34.0-43.8)$ & $38.9(25.0-52.8)$ & $41.8(34.2-49 \cdot 3)$ & $35.0(27.8-42.2)$ & 0.224 \\
\hline Palliative surgery & $19.7(18.0-21.4)$ & $16.6(14.5-18.6)$ & $19.7(17.1-22.3)$ & $21.2(18.1-21.4)$ & 0.078 \\
\hline None & $12.1(11.2-13.1)$ & $9.9(6.3-13.5)$ & $11.5(9.6-13.5)$ & $13.2(10.9-15.6)$ & $0.019^{\mathrm{a}}$ \\
\hline
\end{tabular}

Values are presented as number (\%) or median (range).

OS, overall survival.

${ }^{\mathrm{a}} \mathrm{p}<0.05$. 
curative or palliative resection, no significant differences in OS were observed with respect to primary tumor location ( $p=0.224$ and $p=0.078$, respectively).

\section{Difference in the numbers and pattern of metastatic sites}

The number and pattern of metastatic sites were associated with survival. In patients with a single metastatic site, the median OS differed according to the metastatic site. The median OS was 19.8 (95\% CI, 17.5 to 22.1 ), 27.8 (95\% CI, 23.2 to 32.4 ), 18.0 (95\% CI, 11.0 to 25.0 ), 24.9 (95\% CI, 21.5 to 28.3 ), 4.1 (95\% CI, 2.5 to 5.7 ), and 28.4 months (95\% CI, 26.5 to 30.3) months for patients with liver, lung, peritoneal, extra-regional lymph node, bone and ovarian metastases alone, respectively $(p=0.031)$. The OS of a patient with brain metastasis alone was 15.7 months. A comparison of the different metastatic sites revealed that the survival of patients with peritoneal metastasis was significantly poorer than the survival of patients with liver $(p<0.05)$, lung $(p=0.002)$, or extra-regional lymph node $(p=0.001)$ metastases. In contrast, no signif-

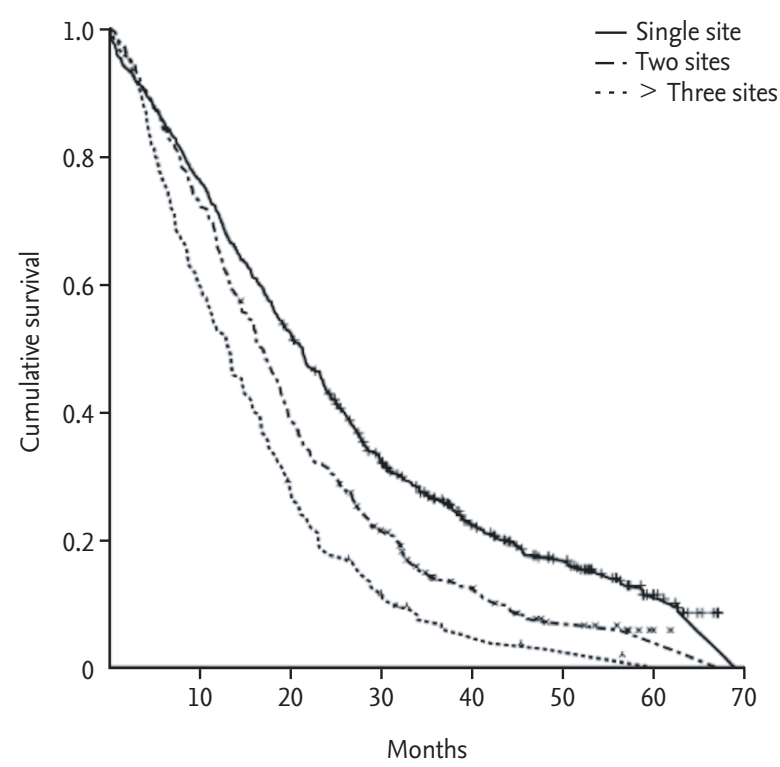

\begin{tabular}{lccc}
\hline No. of metastatic site(s) & Median OS, mon & $95 \% \mathrm{Cl}$ & p value \\
\hline Single & 21.2 & $19.2-23.2$ & \\
Two sites & 16.7 & $15.0-18.4$ & \\
$>$ Three sites & 13.1 & $10.8-15.4<0.001^{a}$ \\
\hline
\end{tabular}

Figure 3. Kaplan-Meier curves of overall survival (OS) according to the number of metastatic sites. CI, confidence interval. ${ }^{\mathrm{a}} \mathrm{p}<0.05$. icant differences were observed for patients with bone or ovarian metastases $(p=0.481$ and $p=0.124$, respectively).

The number of metastatic sites exhibited a statistically significant effect on survival. The median OS of patients with a single metastatic site, including those with a solitary metastasis, was significantly longer than that of patients with multiple metastatic sites (21.2 months [95\% CI, 19.2 to 23.2 ] vs. 16.7 months [ $95 \%$ CI, 15.0 to 18.4] or 13.1 months [ $95 \%$ CI, 10.8 to 15.4 ] for 2 and $\geq 3$ metastatic sites, respectively; $p<0.001$ ) (Fig. 3).

\section{Prognostic factors}

In the univariate analysis, age, the presence of an underlying disease, Eastern Cooperative Oncology Group (ECOG) performance status (PS), the number of metastatic sites, the use of molecularly targeted agents, surgical resection, and primary tumor location correlated with OS. Due to the extent of missing data for the ECOG PS, the use of molecularly targeted agents, and KRAS mutation status, these factors were excluded from the multivariate analysis. In the multivariate analysis, the number of metastatic sites, surgical resection (curative and palliative), and primary tumor location were associated with OS (Table 9).

\section{DISCUSSION}

In this study, we demonstrate that the primary tumor location influences the metastatic sites and prognosis of patients with mCRC. The pattern of metastasis differed according to primary tumor location. Right colon cancer metastasized more frequently to the peritoneum and ovaries and left colon and rectal cancer metastasized more frequently to the lungs. This difference was also reported in recurrence patterns after curative resection or the initial presentation of metastatic disease. Colon cancer patients presented more frequently with intra-abdominal metastases, including peritoneal, omental, and ovarian metastases. Left colon cancer patients were associated with an increased risk of metastatic spread to the liver, whereas rectal cancer patients were associated with an increased risk of local recurrence and metastatic spread to the extra-abdominal sites, including the lungs and brain [13-16]. These differences affected the initial evaluation of CRC and clinical practice. Although lim- 
Table 9. Univariate and multivariate analyses of overall survival in metastatic colorectal cancer

\begin{tabular}{|c|c|c|c|c|}
\hline \multirow{2}{*}{ Characteristic } & \multicolumn{2}{|c|}{ Univariate analysis } & \multicolumn{2}{|c|}{ Multivariate analysis } \\
\hline & $\operatorname{HR}(95 \% \mathrm{CI})$ & $p$ value & $\mathrm{HR}(95 \% \mathrm{CI})$ & $p$ value \\
\hline \multicolumn{5}{|l|}{ Age, yr } \\
\hline$\leq 65$ & 1 & & 1 & \\
\hline$>65$ & $1.39(1.23-1.58)$ & $<0.001^{\mathrm{a}}$ & $1.06(0.93-1.20)$ & 0.394 \\
\hline \multicolumn{5}{|l|}{ Underlying disease } \\
\hline Absent & 1 & & - & \\
\hline Present & $1.03(0.91-1.17)$ & 0.646 & - & - \\
\hline \multicolumn{5}{|c|}{ Primary tumor location } \\
\hline Right colon & 1 & & 1 & \\
\hline Left colon & $0.80(0.68-0.94)$ & $0.007^{\mathrm{a}}$ & $0.81(0.68-0.95)$ & $0.010^{\mathrm{a}}$ \\
\hline Rectum & $0.76(0.64-0.90)$ & $0.001^{\mathrm{a}}$ & $0.78(0.64-0.90)$ & $0.001^{\mathrm{a}}$ \\
\hline \multicolumn{5}{|l|}{ Metastases } \\
\hline Single & 1 & & 1 & \\
\hline Multiple & $1.52(1.34-1.73)$ & $<0.001^{\mathrm{a}}$ & $1.52(1.33-1.72)$ & $<0.001^{\mathrm{a}}$ \\
\hline \multicolumn{5}{|l|}{ Surgery } \\
\hline None & 1 & & 1 & \\
\hline Curative surgery & $0.22(0.18-0.27)$ & $<0.001^{\mathrm{a}}$ & $0.24(0.20-0.29)$ & $<0.001^{\mathrm{a}}$ \\
\hline Palliative surgery & $0.58(0.50-0.67)$ & $<0.001^{\mathrm{a}}$ & $0.58(0.50-0.67)$ & $<0.001^{\mathrm{a}}$ \\
\hline
\end{tabular}

HR, hazard ratio; CI, confidence interval.

$\mathrm{a} p<0.05$.

ited evidence exists regarding the pattern of spread of CRCs, there are several potential explanations for the differences in metastatic patterns, including differences in tumor genetics, biology, and histology [16,17], as well as, anatomically distinct and discrete lymphatic and vascular drainage regions [18].

We have previously established that chemotherapy with or without molecularly targeted agents is associated with prolonged survival in patients with mCRC [812]. In this study, we also demonstrate that the survival of patients with mCRC differs according to the primary tumor location, with a median OS of 13.7, 18.0, and 19.9 months for the right colon, the left colon, and the rectum, respectively. Moreover, in patients who received chemotherapy with molecularly targeted agents the survival benefits were more pronounced for those with tumors originating in the left colon and rectum compared to those with tumors originating in the right colon (Table 7). Although controversial, several studies [19-21] have shown that there may be a survival difference between patients with left and right colon cancer. Meguid et al. [19]. and Benedix et al. [20] reported that patients with right colon cancer had a poorer prognosis than patients with left colon cancer. However, Weiss et al. [21] reported no significant differences in survival between patients with right and left colon cancer. Because these studies focused mainly on early-stage colon cancer, rectal cancer was excluded, due to the different treatments for localized disease. Patients with mCRC were relatively few in number and the pathological information on the tumors was limited. Thus, the influences of the primary tumor location on the clinical outcomes of patients with $\mathrm{mCRC}$ remains controversial.

More recently, responses to chemotherapy with molecularly targeted agents have been shown to differ according to primary tumor location. The addition of bevacizumab to combination chemotherapy for mCRC could benefit patients with primary left colon tumors $[22,23]$. In addition, the anti-EGFR agent, cetuximab, could be beneficial in the treatment of patients with primary left colon tumors $[24,25]$. In this study, KRAS genetic testing was performed in selected mCRC patients, due to the limited coverage of national insurance reimbursement. KRAS mutation status differed according to 
the primary tumor location. KRAS gene mutations occurred more frequently in patients with right colon cancer. In patients, whose tumors expressed the wild-type KRAS gene, the prognosis was significantly better for left colon and rectal cancer patients than right colon cancer patients. These findings were consistent with those of recently published studies. In the NCIC-CTC-CO.17 trial [26], the efficacy of cetuximab in chemorefractory mCRC patients was significantly higher for those with tumors originating in the left colon. The median PFS for cetuximab-treated patients with primary tumors originating in the left and right colon was 5.4 and 1.9 months, respectively $(p=0.002)$. An analysis of the AIO KRK-0104 trial comparing first-line therapies using cetuximab, capecitabine, and irinotecan with cetuximab, capecitabine, and oxaliplatin [24] reported that patients with primary tumors originating in the left colon had a longer OS and PFS compared to patients whose primary tumors had originated in the right colon.

$\mathrm{CRC}$ is a heterogeneous disease with respect to molecular carcinogenesis and morphological multistep pathways. Molecular alterations in CRC may exert their effects through three major pathways: MSI, chromosomal instability (CIN), and CpG island methylator phenotype (CIMP). These pathways illustrate alterations of genes and critical pathways, including defects of DNA mismatch repair genes, APC gene with Wnt signaling activation, TP53 gene with inactivation of $\mathrm{p}_{53}$ pathway, RAS and BRAF gene with mitogen-activated protein kinase signaling pathway, $\mathrm{PI}_{3} \mathrm{KCA}$ gene with $\mathrm{PIK}_{3}$ signaling, inactivation of transforming growth factor $\beta$ pathway, epithelial-to-mesnechymal transition genes and MrC gene amplification, and among others [27,28]. Moreover, these genetic alterations affect morphological multistep pathways, such as the classic adenoma-carcinoma and serrated neoplasia pathways. These alterations can occur individually or in combination, resulting in the growth of tumors with different clinical and morphological characteristics [6,29-31]. Although CIN predominantly occurs in sporadic tumors, irrespective of their anatomical site, sporadic tumors harboring MSIs are localized, especially in the proximal colon $[6,32,33]$. The majority of CIMP-high tumors are associated with MSI, the proximal colon, and KRAS and BRAF mutation [34]. The clinical characteristics of CIMP CRC patients have been compared to those of CRC patients with MSI. The prog- nosis of CRC patients with MSI-high tumors is more favorable compared to the prognosis of CRC patients with CIN [35]. However, the CIMP-high tumors are associated with reduced colon cancer mortality rates. Additionally, BRAF mutation status is frequently identified in tumors with the CIMP-high tumors as being associated with a high colon cancer specific mortality $[36,37]$. In the present study, although the molecular characteristics of CRC (e.g., BRAF mutation, CIMP, and MSI status) could not be evaluated, the prognosis of patients with right colon cancer was poorer compared to that of patients with left colon or rectal cancer. It may be related to these molecular alterations and clinical characteristics that, at the time of initial diagnosis, metastatic right colon cancer is associated with a greater tumor burden than left colon or rectal cancer.

Surgical resection is the treatment of choice for patients with resectable mCRC [38]. However, palliative resection of the primary tumor in $\mathrm{mCRC}$ is controversial. Although selection bias was present, several retrospective analyses [39,40] have demonstrated that palliative resection of the primary tumor is associated with improved survival, especially in relatively young patients with a good PS and tumors that were not poorly differentiated. Our study also revealed that tumor resection, whether curative or palliative, improved the survival of patients with primary tumors at any location. Clinical trials are needed to validate these finding.

\section{KEY MESSAGE}

1. Primary tumor location influences the metastatic sites and prognosis of patients with metastatic colorectal cancer.

2. The effect of chemotherapy with molecularly targeted agents is more pronounced in primary left side tumor location.

3. Surgical resection of the tumor (curative or palliative) may be influenced the survival.

\section{Conflict of interest}

No potential conflict of interest relevant to this article was reported. 


\section{Acknowledgments}

The study was partially supported by the Korean Cancer Study Group (KCSG) and the KCSG Data Center (Hyeimi Park).

\section{REFERENCES}

1. Torre LA, Bray F, Siegel RL, Ferlay J, Lortet-Tieulent J, Jemal A. Global cancer statistics, 2012. CA Cancer J Clin 2015; 65:87-108.

2. Jung KW, Won YJ, Kong HJ, et al. Cancer statistics in Korea: incidence, mortality, survival, and prevalence in 2012. Cancer Res Treat 2015;47:127-141.

3. Greystoke A, Mullamitha SA. How many diseases are colorectal cancer? Gastroenterol Res Pract 2012;2012: 564741 .

4. Bertario L, Russo A, Sala P, et al. Survival of patients with hereditary colorectal cancer: comparison of HNPCC and colorectal cancer in FAP patients with sporadic colorectal cancer. Int J Cancer 1999;80:183-187.

5. Pino MS, Chung DC. The chromosomal instability pathway in colon cancer. Gastroenterology 2010;138:2059-2072.

6. Markowitz SD, Bertagnolli MM. Molecular origins of cancer: molecular basis of colorectal cancer. N Engl J Med 2009;361:2449-2460.

7. Sauer R, Becker H, Hohenberger W, et al. Preoperative versus postoperative chemoradiotherapy for rectal cancer. N Engl J Med 2004;351:1731-1740.

8. Tournigand C, Andre T, Achille E, et al. FOLFIRI followed by FOLFOX6 or the reverse sequence in advanced colorectal cancer: a randomized GERCOR study. J Clin Oncol 2004;22:229-237.

9. Hurwitz H, Fehrenbacher L, Novotny W, et al. Bevacizumab plus irinotecan, fluorouracil, and leucovorin for metastatic colorectal cancer. N Engl J Med 2004;350:23352342.

10. Saltz LB, Clarke S, Diaz-Rubio E, et al. Bevacizumab in combination with oxaliplatin-based chemotherapy as first-line therapy in metastatic colorectal cancer: a randomized phase III study. J Clin Oncol 2008;26:2013-2019.

11. Van Cutsem E, Kohne CH, Hitre E, et al. Cetuximab and chemotherapy as initial treatment for metastatic colorectal cancer. N Engl J Med 2009;360:1408-1417.

12. Douillard JY, Oliner KS, Siena S, et al. PanitumumabFOLFOX 4 treatment and RAS mutations in colorectal cancer. N Engl J Med 2013;369:1023-1034.

13. Augestad KM, Bakaki PM, Rose J, et al. Metastatic spread pattern after curative colorectal cancer surgery: a retrospective, longitudinal analysis. Cancer Epidemiol 2015;39: 734-744.

14. Amri R, Bordeianou LG, Sylla P, Berger DL. Variations in metastasis site by primary location in colon cancer. J Gastrointest Surg 2015;19:1522-1527.

15. Riihimaki M, Hemminki A, Sundquist J, Hemminki K. Patterns of metastasis in colon and rectal cancer. Sci Rep 2016;6:29765.

16. Hugen N, van de Velde CJ, de Wilt JH, Nagtegaal ID. Metastatic pattern in colorectal cancer is strongly influenced by histological subtype. Ann Oncol 2014;25:651-657.

17. Sun C, Zargham R, Shao Q, et al. Association of CD98, integrin $\beta 1$, integrin $\beta 3$ and Fak with the progression and liver metastases of colorectal cancer. Pathol Res Pract 2014;210:668-674.

18. Li FY, Lai MD. Colorectal cancer, one entity or three. J Zhejiang Univ Sci B 2009;10:219-229.

19. Meguid RA, Slidell MB, Wolfgang CL, Chang DC, Ahuja N. Is there a difference in survival between right- versus leftsided colon cancers? Ann Surg Oncol 2008;15:2388-2394.

20. Benedix F, Kube R, Meyer F, et al. Comparison of 17,641 patients with right- and left-sided colon cancer: differences in epidemiology, perioperative course, histology, and survival. Dis Colon Rectum 2010;53:57-64.

21. Weiss JM, Pfau PR, O'Connor ES, et al. Mortality by stage for right- versus left-sided colon cancer: analysis of surveillance, epidemiology, and end results: medicare data. J Clin Oncol 2011;29:4401-4409.

22. Boisen MK, Johansen JS, Dehlendorff C, et al. Primary tumor location and bevacizumab effectiveness in patients with metastatic colorectal cancer. Ann Oncol 2013;24:25542559 .

23. Loupakis F, Yang D, Yau L, et al. Primary tumor location as a prognostic factor in metastatic colorectal cancer. J Natl Cancer Inst 2015;107.

24. von Einem JC, Heinemann V, von Weikersthal LF, et al. Left-sided primary tumors are associated with favorable prognosis in patients with KRAS codon 12/13 wild-type metastatic colorectal cancer treated with cetuximab plus chemotherapy: an analysis of the AIO KRK-0104 trial. J Cancer Res Clin Oncol 2014;140:1607-1614.

25. Wang F, Bai L, Liu TS, et al. Right-sided colon cancer and left-sided colorectal cancers respond differently to cetux- 
imab. Chin J Cancer 2015;34:384-393.

26. Brule SY, Jonker DJ, Karapetis CS, et al. Location of colon cancer (right-sided versus left-sided) as a prognostic factor and a predictor of benefit from cetuximab in NCIC CO.17. Eur J Cancer 2015;51:1405-1414.

27. Cancer Genome Atlas Network. Comprehensive molecular characterization of human colon and rectal cancer. Nature 2012;487:330-337.

28. Fearon ER. Molecular genetics of colorectal cancer. Annu Rev Pathol 2011;6:479-507.

29. Yamauchi M, Morikawa T, Kuchiba A, et al. Assessment of colorectal cancer molecular features along bowel subsites challenges the conception of distinct dichotomy of proximal versus distal colorectum. Gut 2012;61:847-854.

30. Bae JM, Kim JH, Kang GH. Molecular subtypes of colorectal cancer and their clinicopathologic features, with an emphasis on the serrated neoplasia pathway. Arch Pathol Lab Med 2016;140:406-412.

31. Shia J, Schultz N, Kuk D, et al. Morphological characterization of colorectal cancers in The Cancer Genome Atlas reveals distinct morphology-molecular associations: clinical and biological implications. Mod Pathol 2017;30:599609.

32. Fearon ER, Vogelstein B. A genetic model for colorectal tumorigenesis. Cell 1990;61:759-767.

33. Young J, Simms LA, Biden KG, et al. Features of colorectal cancers with high-level microsatellite instability occur- ring in familial and sporadic settings: parallel pathways of tumorigenesis. Am J Pathol 2001;159:2107-2116.

34. Ang PW, Loh M, Liem N, et al. Comprehensive profiling of DNA methylation in colorectal cancer reveals subgroups with distinct clinicopathological and molecular features. BMC Cancer 2010;10:227.

35. Popat S, Hubner R, Houlston RS. Systematic review of microsatellite instability and colorectal cancer prognosis. J Clin Oncol 2005;23:609-618.

36. Ogino S, Nosho K, Kirkner GJ, et al. CpG island methylator phenotype, microsatellite instability, BRAF mutation and clinical outcome in colon cancer. Gut 2009;58:90-96.

37. Popovici V, Budinska E, Tejpar S, et al. Identification of a poor-prognosis BRAF-mutant-like population of patients with colon cancer. J Clin Oncol 2012;30:1288-1295.

38. Schmoll HJ, Van Cutsem E, Stein A, et al. ESMO Consensus guidelines for management of patients with colon and rectal cancer: a personalized approach to clinical decision making. Ann Oncol 2012;23:2479-2516.

39. Park JH, Kim TY, Lee KH, et al. The beneficial effect of palliative resection in metastatic colorectal cancer. $\mathrm{Br} \mathrm{J}$ Cancer 2013;108:1425-1431.

40. Gulack BC, Nussbaum DP, Keenan JE, et al. Surgical resection of the primary tumor in stage IV colorectal cancer without metastasectomy is associated with improved overall survival compared with chemotherapy/radiation therapy alone. Dis Colon Rectum 2016;59:299-305. 